Review began 01/26/2022 Review ended 02/02/2022 Published 02/07/2022

\section{(c) Copyright 2022}

Iqbal et al. This is an open access article distributed under the terms of the Creative Commons Attribution License CC-BY 4.0., which permits unrestricted use, distribution, and reproduction in any medium, provided the original author and source are credited.

\title{
Spectrum of Dyslipidemias in Treatment-Naïve Human Immunodeficiency Virus-Infected Patients Presenting to an HIV Clinic of a Tertiary Care Hospital
}

\author{
Sadaf Iqbal ${ }^{1}$, Sadia Salman ${ }^{1}$, Mehwish Akhtar ${ }^{2}$, Amanullah Bhalli ${ }^{1}$, Javeid Iqbal ${ }^{3}$, Ismat Ullah ${ }^{4}$ \\ 1. Department of Internal Medicine, Diabetes and Endocrinology, Jinnah Hospital, Allama Iqbal Medical College, \\ Lahore, PAK 2. Department of Epidemiology and Public Health, Jinnah Hospital, Allama Iqbal Medical College, Lahore, \\ PAK 3. Department of Medicine, Fatima Memorial Hospital College of Medicine \& Dentistry, Lahore, PAK 4. \\ Department of Family Medicine, Allama Iqbal Medical College, Lahore, PAK
}

Corresponding author: Sadaf Iqbal, iqbalian205@gmail.com

\section{Abstract}

\section{Introduction}

HIV/AIDS is a major communicable disease worldwide, especially in developing countries where disease prevalence is over 90\%. The National AIDS Control Programme of Pakistan reported around 160,000 HIV cases $(140,000-190,000)$ with a 5\% prevalence among traditional risks groups. HIV infection is thought to affect lipids metabolism adversely, thus resulting in increased morbidity and mortality. The aim of the study was to find out the frequency and types of dyslipidemia in patients with HIV not taking anti-retroviral therapy, presenting to an HIV clinic at a tertiary care hospital.

\section{Methods}

This cross-sectional study was conducted at the HIV clinic of Jinnah Hospital from January 2020 to July 2020. A total of 280 treatment-naïve patients, fulfilling the inclusion protocol, were included through nonprobability consecutive sampling after informed consent. Blood samples of $5 \mathrm{~mL}$ were taken using aseptic measures and following standard procedure after ensuring overnight fasting by a nurse and were sent immediately to the pathology laboratory of Allama Iqbal Medical College. The results of the lipid profile were collected the next day and noted in the proforma. Dyslipidemia and type of dyslipidemia were recorded as per operational definition. Data were analyzed by SPSS software, version 27.0 (IBM Corp., Armonk, NY). Cross-tabulation was done to assess the relationship of gender, BMI, and family history on dyslipidemia, and a chi-square test was applied to check statistical significance.

\section{Results}

Among 280 treatment-naïve HIV-infected patients, the majority of patients were females (52\%). The mean duration of HIV was $9.31+2.13$ months. About $55 \%$ of patients had a BMI of more than $25 \mathrm{~kg} \mathrm{~m}^{2}$. A family history of dyslipidemia was found in $62 \%$ of the patients. Dyslipidemia was observed in $70 \%$ of patients with maximum derangement seen in total cholesterol level (62\%). After applying the chi-square test, a significant relation was identified between BMI and family history with dyslipidemia in HIV-infected individuals (pvalue $=0.00$ )

\section{Conclusion}

A considerable proportion of treatment-naïve HIV patients have underlying dyslipidemia with a significant relationship with higher BMI and a family history of dyslipidemia. The findings of this study highlight the importance of early screening for dyslipidemia in HIV patients.

Categories: Endocrinology/Diabetes/Metabolism, HIV/AIDS

Keywords: dyslipidemia, bmi, family history, treatment-naive, hiv infection

\section{Introduction}

Globally, the human immunodeficiency virus (HIV) pandemic has infected 59 million people with 20 million deaths so far. The National AIDS Control Programme reported around 160,000 HIV cases (140,000-190,000) with a prevalence of $0.1 \%$. New HIV-infected people of all ages are reported around 20,000-24,000. The death rate is increasing from 1,400 in 2010 to 6,400 in 2018 [1].

HIV infection causes sickness like mononucleosis with a slew of non-specific symptoms. An estimated 10\% to $60 \%$ of patients with early HIV infection would have no symptoms [2]. In patients with acute symptomatic HIV infection, the average time between HIV exposure and the start of symptoms is two to four weeks; 
however, incubation durations of up to 10 months have been observed [3]. A variety of symptoms and signs that are commonly referred to as acute retroviral syndrome may be detected. In a published series, the most common symptoms include headache, fever, rash, sore throat, arthralgia, myalgia, and lymphadenopathy [46].

Liu et al. found gradual increasing dyslipidemia in treatment-naïve HIV patients. HIV itself and antiretroviral (ART) therapy can cause dyslipidemia, so extra care should be taken by the clinicians to an early screening of dyslipidemia to improve quality of life [7]. Bekolo et al. discovered in a cross-sectional research of 114 participants that the prevalence of dyslipidemia was $70 \%$, while hypercholesterolemia was found in 34 (30\%) individuals [8]. Bourgi et al. observed in a study that patients living with HIV suffer metabolic complications like insulin resistance and increased free fatty acids [9]. Duro et al. concluded that metabolic syndrome is highly likely to occur in HIV-infected patients who are virally repressed than in patients who have an acute infection [10].

Adal et al. concluded in their cross-sectional study that high cholesterol and high triglycerides (TGs) levels were prevalent in ART naïve-infected patients, so lipid levels should be observed regularly in patients whether on or off ART [11]. Souza et al. assessed 190 peer-reviewed scholarly articles. Patients with HIV/AIDS who did not get ART medication had higher levels of total cholesterol, TGs, low-density lipoprotein cholesterol (LDL-c), and high-density lipoprotein cholesterol (HDL-c). Several ART regimens have different effects on lipid metabolism, especially protease inhibitors. As a result, the infection, distinct classes of drugs, and some compounds from the same domain of ART appear to cause differential modifications in lipid metabolism; for example, nevirapine was found to be strongly related with high HDL-c levels, a cardiovascular disease-protective component [12].

As the incidence rate and death rate are increasing in Pakistan because of HIV, according to the latest Joint United Nations Programme on HIV/AIDS (UNAIDS) report, there is a need to address its morbidity and mortality [13]. Lipid disorders are more prevalent among HIV-infected patients whether they are taking ART medications or not. Understanding these dyslipidemias is important to screen or diagnose the cause of lipid disorder, which may lead to life-threatening cardiovascular diseases and will improve the treatment outcomes in HIV-infected patients, thus providing a better quality of life [14].

\section{Materials And Methods}

A cross-sectional study was conducted in the HIV clinic at Jinnah Hospital, Lahore from January 2020 to July 2020. The sample size of 280 cases was estimated with a $95 \%$ confidence interval, $5 \%$ margin of error, and the expected percentage of patients with high total cholesterol levels as $69 \%$.

Treatment-naïve HIV-infected patients for at least six months, aged 20-65 years, were included in the study by non-probability consecutive sampling. Patients with a history of familial dyslipidemia, known dyslipidemia, using lipid-lowering or already on retroviral medication, diabetes, hypertension, or metabolic syndrome were excluded from the study. Informed consent was obtained from them before they were included in the study. The researcher took blood samples using aseptic precautions and regular protocol after assuring eight hours of overnight fasting and promptly submitted them to the pathology laboratory of Allama Iqbal Medical College, Lahore. A pre-designed proforma was used to record the study variables and the demographic information, as well as reports of lipid profiles. The data's confidentiality was ensured. Dyslipidemia and type of dyslipidemia were documented in accordance with the operational criteria.

SPSS software version 27.0 (IBM Corp., Armonk, NY) was used to enter and analyze data. Age was summarized numerically as mean and standard deviation (SD). Qualitative attributes such as gender, dyslipidemia, and its type, i.e., high cholesterol, high TG, low high-density lipoprotein (HDL), high lowdensity lipoprotein (LDL), and high very-low-density lipoprotein (VLDL) levels, were provided as frequency and percentages. To account for impact modifiers, data were stratified by gender, BMI, and family history of dyslipidemia. The post-stratification chi-square test was used with p-values less than 0.05 as statistically significant.

\section{Results}

Table 1 displays the descriptive statistics of the patient profile of $280 \mathrm{HIV}$-infected individuals having no ART treatment and aged 25 to 60 years old with a mean of $41.72 \pm 10.47$ years. The mean height, weight, and BMI were $166.19+8.37 \mathrm{~cm}, 78.06+10.32 \mathrm{~kg}$, and $25.15+2.84 \mathrm{~kg} \mathrm{~m}^{2}$, respectively. The mean duration of HIV infection was $9.31+2.13$ months. There were 134 (48\%) males and 136 (52\%) females. The majority of patients $(154,55 \%)$ had a BMI of more than $25 \mathrm{~kg} \mathrm{~m}^{2}$. There were $173(62 \%)$ patients who had a family history of dyslipidemia. 


\section{Cureus}

\begin{tabular}{|c|c|c|c|}
\hline \multicolumn{2}{|l|}{ Variables } & Mean & Std. deviation \\
\hline \multicolumn{2}{|l|}{ Age } & 41.72 & 10.47 \\
\hline \multicolumn{2}{|l|}{ Height } & 166.19 & 8.37 \\
\hline \multicolumn{2}{|l|}{ Weight } & 78.06 & 10.32 \\
\hline \multicolumn{2}{|l|}{ BMI } & 25.15 & 2.84 \\
\hline \multicolumn{2}{|l|}{ Duration of HIV } & 9.31 & 2.13 \\
\hline \multicolumn{2}{|l|}{ Variables } & Frequency $(n=280)$ & Percent $(100 \%)$ \\
\hline \multirow[t]{2}{*}{ Gender } & Male & 134 & $48 \%$ \\
\hline & Female & 146 & $52 \%$ \\
\hline \multirow[t]{2}{*}{ BMI } & $\mathrm{BMI}>25$ & 154 & $55 \%$ \\
\hline & $\mathrm{BMI}<25$ & 126 & $45 \%$ \\
\hline \multirow[t]{2}{*}{ Family history of dyslipidemia } & Yes & 173 & $62 \%$ \\
\hline & No & 107 & $38 \%$ \\
\hline
\end{tabular}

TABLE 1: Descriptive statistics of patient profile $(n=280)$.

Table 2 displays the frequency distribution of dyslipidemia and its spectrum. It was found that 196 individuals (70\%) had dyslipidemia, 173 (62\%) patients had cholesterol levels greater than $200 \mathrm{mg} / \mathrm{dl}, 140$ (50\%) had TG levels greater than $150 \mathrm{mg} / \mathrm{dl}, 170$ (61\%) had LDL levels less than $130 \mathrm{mg} / \mathrm{dl}$, and 207 (74\%) patients had HDL levels greater than $35 \mathrm{mg} / \mathrm{dl}$.

\begin{tabular}{|c|c|c|}
\hline Dyslipidemia & Frequency $(n=280)$ & Percent, $\mathbf{1 0 0 \%}$ \\
\hline Yes & 196 & $70 \%$ \\
\hline No & 84 & $30 \%$ \\
\hline \multicolumn{3}{|l|}{ Cholesterol level } \\
\hline$>200$ mg/dl & 173 & $62 \%$ \\
\hline$<200$ mg/dl & 107 & $38 \%$ \\
\hline \multicolumn{3}{|l|}{ Triglyceride level } \\
\hline >150 mg/dl & 140 & $50 \%$ \\
\hline$<150 \mathrm{mg} / \mathrm{dl}$ & 140 & $50 \%$ \\
\hline \multicolumn{3}{|l|}{ LDL Level } \\
\hline$>130$ mg/dl & 110 & $39 \%$ \\
\hline$<130$ mg/dl & 170 & $61 \%$ \\
\hline \multicolumn{3}{|l|}{ HDL Level } \\
\hline >35 mg/dl & 207 & $74 \%$ \\
\hline$<35$ mg/dl & 73 & $26 \%$ \\
\hline
\end{tabular}

\section{TABLE 2: Frequency distribution of dyslipidemia and its spectrum.}

LDL, low-density lipoprotein; HDL, high-density lipoprotein. 
Table 3 presents the stratification of effect modifiers. There is a significant relationship between BMI, a familial history of dyslipidemia, and dyslipidemia in treatment-naïve HIV-infected patients with p-value = 0.000. On the other hand, gender had no significant relation with dyslipidemia with a p-value of 0.754.

\begin{tabular}{|c|c|c|c|c|c|}
\hline \multirow{2}{*}{ BMI vs. dyslipidemia } & & \multicolumn{2}{|c|}{ Dysiliaemia } & \multirow{2}{*}{ Total } & \multirow[t]{2}{*}{ P-value } \\
\hline & & Yes & No & & \\
\hline \multirow{2}{*}{ BMI } & $<25 \mathrm{~kg} \mathrm{~m}^{2}$ & 55 & 71 & 126 & \\
\hline & $>25 \mathrm{~kg} \mathrm{~m}^{2}$ & 141 & 13 & 154 & $p=0.00$ \\
\hline Total & & 196 & 84 & 280 & \\
\hline \multicolumn{2}{|l|}{ Family history of dyslipidemia vs. dyslipidemia } & Yes & No & Total & \\
\hline \multirow{2}{*}{ Family history of dyslipidemia } & Yes & 149 & 24 & 173 & \\
\hline & No & 47 & 60 & 107 & $p=0.00$ \\
\hline Total & & 196 & 84 & 280 & \\
\hline Gender vs. dyslipidemia & & Yes & No & Total & \\
\hline \multirow[t]{2}{*}{ Gender } & Male & 95 & 39 & 134 & \\
\hline & Female & 101 & 45 & 146 & $p=0.75$ \\
\hline Total & & 196 & 84 & 280 & \\
\hline
\end{tabular}

TABLE 3: Stratification of effect modifiers $(n=280)$.

\section{Discussion}

Our study found an overall higher prevalence (70\%) of dyslipidemias in treatment-naïve HIV patients as compared to the general population. Prevalence of dyslipidemias was much more in those HIV treatmentnaïve patients who were having a family history of dyslipidemias ( $\mathrm{p}$-value $=0.00)$ or BMI greater than $25(\mathrm{p}$ value $=0.00)$.

Dyslipidemias are a diverse group of lipid metabolism disorders characterized by abnormal levels of various lipid entities in blood. They have gained medical attention due to their pivotal role in the atherosclerosis process. Cholesterol and TGs are the major lipid formulations that are often found deranged in dyslipidemias and form the basis of dyslipidemias classification. Recent literature revealed that the occurrence of opportunistic infections also had an effect on lipid markers and HIV infection is correlated with dyslipidemia, which worsens as the disease develops. In the early stages of HIV infection, HDL-c was found to be lower, with raised TG and atherogenicity index [15].

Teto et al. also said that HIV infection has been shown to have an effect on lipid profile and antioxidant defense. According to the findings, HIV infection is connected to a substantial drop in total antioxidant ability (TAA), LDL-c, HDL-c, and TC. The findings point to increased oxidative stress and lipid peroxidation in HIV-positive Cameroonian patients, as well as a role for circulating recombinant forms (CRFs) in total cholesterol (TC) and malondialdehyde (MDA) levels [16]. Liu et al. found gradually increasing dyslipidemia in treatment-naïve HIV patients, HIV itself, and ART therapy can cause dyslipidemia, so extra care should be taken by the clinicians to an early screening of dyslipidemia to improve quality of life.

Bekolo et al. conducted a cross-sectional study that included 114 HIV-infected people aged 15 years with 83 (73\%) being females. Dyslipidemia was found in $70 \%$ of people. In our study, the prevalence of dyslipidemias was also $70 \%$ but there was no statistically significant gender difference [17]. Calza et al. concluded in a cross-sectional study that in treatment-naïve HIV patients, mostly TGs were raised (44.3\%) with low HDL (41\%) [18]. Our study showed raised cholesterol (>200 mg/dl) and TGs (>150 mg/dl) levels in $62 \%$ and $50 \%$ of patients, respectively. According to Muhammad et al., the mean HDL level in the HIV-positive group was substantially lower in the HIV-positive group as compared to the normal population, indicating a higher frequency of dyslipidemia among patients with HIV. In our study, 26.1\% of patients were having a serum HDL value $<35 \mathrm{mg} / \mathrm{dl}$. Moreover, an LDL level of $>130 \mathrm{mg} / \mathrm{dl}$ was observed in $39.3 \%$ of patients. Serum lipid abnormalities are prominent amongst treatment-naïve HIV-infected individuals in Nigeria [19]. 
Limitations of the current study are that it is a single-center study done on a limited number of treatmentnaïve patients only. Larger multicenter studies are needed to gain further insight into these dyslipidemias and HIV correlation. The findings of this study highlight the importance of early screening for dyslipidemia in HIV patients and their management to reduce cardiovascular complications [20].

\section{Conclusions}

Lipid abnormalities are prevalent in HIV-infected individuals who have not received treatment. Greater BMI and a family history of dyslipidemia were significantly related to the occurrence of dyslipidemia in treatment-naïve HIV-infected patients. So, clinicians need to focus on early diagnosis and screening of dyslipidemia and its management. This will decrease the risk of cardiovascular problems, thus resulting in better survival as per evidence-based management.

\section{Additional Information \\ Disclosures}

Human subjects: Consent was obtained or waived by all participants in this study. Ethical Review Board Allama Iqbal Medical College Jinnah Hospital, Lahore issued approval Ref no. 38/ERB Dated Nov 1, 2019. This is to state that the research project "Spectrum of Dyslipidemias in Treatment-Naive Human Immunodeficiency Virus-Infected Patients Presenting to HIV Clinic of a Tertiary Care Hospital" by Dr. Sadaf Iqbal, Fellow of Endocrinology, is approved by the Ethical Review Board on November 1, 2019. Animal subjects: All authors have confirmed that this study did not involve animal subjects or tissue. Conflicts of interest: In compliance with the ICMJE uniform disclosure form, all authors declare the following: Payment/services info: All authors have declared that no financial support was received from any organization for the submitted work. Financial relationships: All authors have declared that they have no financial relationships at present or within the previous three years with any organizations that might have an interest in the submitted work. Other relationships: All authors have declared that there are no other relationships or activities that could appear to have influenced the submitted work.

\section{Acknowledgements}

I would like to acknowledge the immense support of my wife, Dr. Ammara, in carrying out this research, who supported me a lot throughout my endocrinology fellowship.

\section{References}

1. National AIDS Control Programme. Publications. (2021). Accessed: December 19, 2021 https://nacp.gov.pk/howwework/publications.html.

2. Panel on Antiretroviral Guidelines for Adults and Adolescents. Guidelines for the use of antiretroviral agents in adults and adolescents with HIV. (2021). https://clinicalinfo.hiv.gov/sites/default/files/guidelines/documents/AdultandAdolescentGL.pdf.

3. Ridzon R, Gallagher K, Ciesielski C, Ginsberg MB, Robertson BJ, Luo CC, DeMaria A Jr: Simultaneous transmission of human immunodeficiency virus and hepatitis $\mathrm{C}$ virus from a needle-stick injury. $\mathrm{N}$ Engl J Med. 1997, 336:919-22. 10.1056/NEJM199703273361304

4. Kared H, Lelièvre JD, Donkova-Petrini V, et al.: HIV-specific regulatory T cells are associated with higher CD4 cell counts in primary infection. AIDS. 2008, 22:2451-60. 10.1097/QAD.0b013e328319edc0

5. Niu MT, Stein DS, Schnittman SM: Primary human immunodeficiency virus type 1 infection: review of pathogenesis and early treatment intervention in humans and animal retrovirus infections. J Infect Dis. 1993, 168:1490-501. 10.1093/infdis/168.6.1490

6. Daar ES, Little S, Pitt J, et al.: Diagnosis of primary HIV-1 infection . Ann Intern Med. 2001, 134:25-9. 10.7326/0003-4819-134-1-200101020-00010

7. Liu D, Zhang X, Kang J, Gao F, He Y, He S: Gradual increasing dyslipidemia in treatment-naive male patients with human immunodeficiency virus and treated with tenofovir plus lamivudine plus efavirenz for 3 years. Diabetol Metab Syndr. 2021, 13:135. 10.1186/s13098-021-00756-y

8. Bekolo CE, Nguena MB, Ewane L, Bekoule PS, Kollo B: The lipid profile of HIV-infected patients receiving antiretroviral therapy in a rural Cameroonian population. BMC Public Health. 2014, 14:236. 10.1186/14712458-14-236

9. Bourgi K, Wanjalla C, Koethe JR: Inflammation and metabolic complications in HIV. Curr HIV/AIDS Rep. 2018, 15:371-81. 10.1007/s11904-018-0411-2

10. Duro M, Manso MC, Barreira S, Rebelo I, Medeiros R, Almeida C: Metabolic syndrome in human immunodeficiency virus-infected patients. Int J STD AIDS. 2018, 29:1089-97. 10.1177/0956462418775188

11. Adal M, Howe R, Kassa D, Aseffa A, Petros B: Malnutrition and lipid abnormalities in antiretroviral naïve HIV-infected adults in Addis Ababa: a cross-sectional study. PLoS One. 2018, 13:e0195942. 10.1371/journal.pone.0195942

12. Souza SJ, Luzia LA, Santos SS, Rondó PH: Lipid profile of HIV-infected patients in relation to antiretroviral therapy: a review. Rev Assoc Med Bras (1992). 2013, 59:186-98. 10.1016/j.ramb.2012.11.003

13. Drechsler H, Ayers C, Cutrell J, Arasaratnam R, Bedimo R: Consistent use of lipid lowering therapy in HIV infection is associated with low mortality. BMC Infect Dis. 2021, 21:150. 10.1186/s12879-021-05787-4

14. Li Y, Wang Z, Xia H, Zhang J: Influence of statin therapy on the incidence of cardiovascular events, cancer, and all-cause mortality in people living with HIV: a meta-analysis. Front Med (Lausanne). 2021, 8:769740. 10.3389/fmed.2021.769740 


\section{Cureus}

15. Hopkins RS, Jajosky RA, Hall PA, et al.: Summary of notifiable diseases--United States, 2003. MMWR Morb Mortal Wkly Rep. 2005, 52:1-85.

16. Teto G, Kanmogne GD, Torimiro JN, et al.: Lipid peroxidation and total cholesterol in HAART-naïve patients infected with circulating recombinant forms of human immunodeficiency virus type- 1 in Cameroon. PLoS One. 2013, 8:e65126. 10.1371/journal.pone.0065126

17. Lo J: Dyslipidemia and lipid management in HIV-infected patients. Curr Opin Endocrinol Diabetes Obes. 2011, 18:144-7. 10.1097/MED.0b013e328344556e

18. Calza L, Colangeli V, Magistrelli E, et al.: Prevalence of metabolic syndrome in HIV-infected patients naive to antiretroviral therapy or receiving a first-line treatment. HIV Clin Trials. 2017, 18:110-7. $10.1080 / 15284336.2017 .1311502$

19. Muhammad S, Sani MU, Okeahialam BN: Prevalence of dyslipidemia among human immunodeficiency virus infected Nigerians. Ann Afr Med. 2013, 12:24-8. 10.4103/1596-3519.108246

20. Maggi P, Di Biagio A, Rusconi S, et al.: Cardiovascular risk and dyslipidemia among persons living with HIV: a review. BMC Infect Dis. 2017, 17:551. 10.1186/s12879-017-2626-Z 